\title{
Successful management of bleeding complications in patients supported with extracorporeal membrane oxygenation with primary respiratory failure.
}

K Lamb

Sw Cowan

N Evans

H Pitcher

T Moritz

Follow this and additional works at: https://jdc.jefferson.edu/cbfp See next page for additional authors

Part of the Surgery Commons

Let us know how access to this document benefits you

\section{Recommended Citation}

Lamb, K; Cowan, Sw; Evans, N; Pitcher, H; Moritz, T; Lazar, M; Hirose, H; and Cavarocchi, Nc, "Successful management of bleeding complications in patients supported with extracorporeal membrane oxygenation with primary respiratory failure." (2012). Department of Cancer Biology Faculty Papers. Paper 61.

https://jdc.jefferson.edu/cbfp/61

This Article is brought to you for free and open access by the Jefferson Digital Commons. The Jefferson Digital Commons is a service of Thomas Jefferson University's Center for Teaching and Learning (CTL). The Commons is a showcase for Jefferson books and journals, peer-reviewed scholarly publications, unique historical collections from the University archives, and teaching tools. The Jefferson Digital Commons allows researchers and interested readers anywhere in the world to learn about and keep up to date with Jefferson scholarship. This article has been accepted for inclusion in Department of Cancer Biology Faculty Papers by an authorized administrator of the Jefferson Digital Commons. For more information, please contact: JeffersonDigitalCommons@jefferson.edu. 


\section{Authors}

K Lamb, Sw Cowan, N Evans, H Pitcher, T Moritz, M Lazar, H Hirose, and Nc Cavarocchi 


\title{
As submitted to:
}

\section{Perfusion}

\section{And later published as:}

\section{Successful management of bleeding complications in patients supported with extracorporeal membrane oxygenation with primary respiratory failure.}

October 26, 2012,

\section{doi:10.1177/0267659112464096}

\begin{abstract}
Background: ECMO is a lifesaving procedure in patients with severe respiratory insufficiency failing conventional support. Bleeding complications are common due to the necessity for anticoagulation and circuit related factors.
\end{abstract}

Methods: A retrospective review was conducted in patients requiring ECMO for respiratory failure from $7 / 2010$ to $6 / 2011$ to identity episodes of major bleeding, bleeding management and outcomes.

Results: Twenty-one patients were supported with ECMO during the study, although 5 experienced massive bleeding related to chest tube insertion, jejunal arterio-venous malformations, distal perfusion cannula dislodgement, and ventricular rupture. Patients required aggressive resuscitation, endoscopic or operative intervention, totaling 28 procedures. There were no instances of dehiscence, infection, or sepsis related to 
interventions. Anticoagulation was stopped 6 hours before and restarted 24 hours after major interventions, with no thrombotic or neurologic complications. All patients weaned off ECMO were discharged.

Conclusions: ECMO bleeding complications can be managed successfully via surgical and endoscopic approaches in this high risk population.

Abstract word count: 148 


\section{INTRODUCTION}

Primary reversible respiratory failure is an indication for ECMO in patients who fail conventional respiratory support measures. ${ }^{1}$ Bleeding complications are common and have been reported in $22-32 \%$ of all ECMO supported patients. ${ }^{2,3} \mathrm{~A}$ high incidence of bleeding can be attributed in part to systemic anticoagulation, circuit induced coagulopathies and systemic disease processes, ultimately leading to an increased mortality in these ECMO supported patients.

This paper represents the largest experience reported in the literature pertaining to patient survival of massive bleeding complications on ECMO and the standardized approach to their management; utilizing an anticoagulation protocol and aggressive surgical management.

\section{METHODS}

We performed an Institutional Review Board (IRB) approved review of adult patients from July 2010 to June 2011 in the Surgical Cardiac Care Unit of Thomas Jefferson University Hospital. Twenty-one patients were supported with ECMO during the study period. Of this population, 5 patients ( 2 females, 3 males) experienced massive bleeding related to pre or post ECMO chest tube insertion, jejunal arteriovenous malformations (AVM), femoral distal limb cannula dislodgement with a superficial femoral artery pseudo aneurysm, or ventricular rupture at the time of Avalon ${ }^{\mathrm{TM}}$ cannula insertion. Massive bleeding was defined by our hospital's Massive Transfusion Protocol of transfusion of 10 units of packed red blood cells (PRBC) over 24 hours. Patient data was reviewed retrospectively from a prospective data collecting system. Demographic data, indications and duration for ECMO, occurrence of cerebral events, heparin 
protocols, bleeding complications and their management, and short and long-term outcomes were reviewed.

\section{VA-ECMO/VV-ECMO Cannulation Method:}

Twenty-one patients were peripherally cannulated via percutaneous insertion of the ECMO cannula using the Seldinger technique. All patients on VV-ECMO were cannulated via the internal jugular vein using the Avalon ${ }^{\mathrm{TM}}$ Elite Dual Lumen bicaval cannula (Avalon Laboratories, Rancho Dominquez, CA). Percutaneous femoral cannulation was the preferred placement in all patients on VA-ECMO. Distal arterial percutaneous perfusion catheters $(5 \mathrm{Fr})$ were placed in all patients with peripheral femoral cannulation.

The heart and lung support (HLS) cannulae (bio-compatible polyurethane, Maquet Cardiovascular LLC, San Jose, CA) were connected to a closed crystalloid primed circuit $(\sim 500 \mathrm{ml})$; both cannulae and tubing have Bioline heparin coating. The circuitry for ECMO consisted of the Quadrox-D diffusion membrane hollow-fiber oxygenator (Maquet Cardiovascular LLC, San Jose, CA) and a Rotaflow centrifugal pump (Maquet Cardiovascular LLC, San Jose, CA) with a heater exchanger.

\section{Heparin Protocol}

Whether VV or VA ECMO circuit was used, a bolus of heparin $5000-7500$ units was given just after placement of the venous and/or arterial guide wires. No additional heparin was given for 24 hours after ECMO was initiated unless clots were observed in the circuit. After 24 hours, a heparin infusion was started with a Prothrombin time (PTT) 
therapeutic goal of 40-45 seconds; on day \#2 onwards, PTT therapeutic goal was 45-55 seconds.

The ECMO circuit was continuously monitored for thrombi and fibrin formation by visual inspection. Provided oxygenation and flow were adequate, circuit exchange was not necessary. If thrombi are noted in either the ECMO oxygenator or the patient developed hypoxia, the post-oxygenator oxygenation was evaluated. If the oxygenator was suspected to be the source of the problem, the circuit was exchanged at the bedside. No additional heparin was used for circuit exchanges.

All patients with decreased platelets were evaluated for heparin induced thrombocytopenia (HIT) and anticoagulation was switched to Argatroban if the HIT panel was positive. One patient was suspected to have HIT and temporarily (<24 hours) required Argatroban. Argatroban was discontinued within 24 hours when the HIT panel (platelet factor-4 and serotonin release assay) was negative.

\section{Surgical Procedures}

Major and minor procedures were classified according to urgency of the procedure. For minor procedures, heparin was stopped 6 hours prior to the procedure and was restarted 6 hours after the procedure if there was no evidence of surgical site bleeding. For major procedures (non-emergent), heparin was stopped 6 hours prior to the procedure to minimize intra-operative bleeding. Heparin was routinely restarted 24 hours post-procedure unless bleeding or the bleeding risk is excessively high due to extensiveness of the procedure, then anticoagulation was held an additional 24 hours and re-evaluated .

A summary of patient and ECMO data are found in Table 1. 


\section{CASE REPORTS}

\section{Case \#1: Aspiration pneumonia/ ARDS}

A 17 year-old-male was admitted with a cervical injury and subsequently suffered an aspiration event. He developed pneumonia progressing to adult respiratory distress syndrome (ARDS), and a right-sided pneumothorax. A chest tube was placed at the bedside, and subsequently VV ECMO using the Avalon Cannula was initiated. Heparin was administered at the time of ECMO cannula insertion and a heparin drip was started 24 hours after initiating ECMO titrating PTT to 45-55. He developed a right-sided hemothorax after ECMO cannulation, prompting a series of three right video assisted thoracoscopies (VATS) with washout of the right chest and multiple chest tube placements. Anticoagulation was held 6 hours prior to the VATS and restarted 24 hours after surgery, with adjusted PTT of 40-45. Over the subsequent 2 days, blood accumulated in the right chest, despite low therapeutic PTTs, requiring definitive hemostasis via a right posterior-lateral thoracotomy and control of chest wall hemorrhage. Bleeding was secondary to muscle and chest wall perforators. His resuscitation involved transfusion of 39 units of $\mathrm{PRBC}$ and 25 units of platelets. ECMO was weaned after 10 days, requiring a total of 2 ECMO circuit exchanges. He was later discharged to rehab, able to tracheal collar and tolerate oral intake.

\section{Case \#2: Aspiration pneumonia/ ARDS}

A 21 year-old-female with an inhalational overdose of formaldehyde developed progressive respiratory acidosis and hypoxia. VA ECMO was initiated for an atrial septal defect and acidosis. Several bronchoscopies were performed for endobronchial clot and multiple chest tubes placed for pneumothoraces and loculated empyema. Two left-sided thoracotomies were necessary with clot removal and chest tube placements. An 
exploratory laparotomy was performed for free intraperitoneal air, ultimately attributed to pneumothoraces. She received a right VATS with chest tube placements for a persistent pneumothorax. A tracheostomy and percutaneous gastrostomy tube were placed while on ECMO. For surgical interventions, anticoagulation was held 6-24 hours based on the extensiveness of surgery, with a goal PTT 40-45 due to bleeding complications. Her length of stay on ECMO was 26 days, with 3 ECMO oxygenator exchanges and transfusion of 96 units of PRBC with 130 units of platelets during her resuscitation. Upon discharge to rehab, this patient was trach collaring and tolerating tube feeds. She had no oxygen requirements at her 2 months office visit.

\section{Case \#3: Empyema/ ARDS}

A 47 year-old-male presented with an empyema warranting bedside chest tube placement. An expanding hemothorax developed, progressing to ARDS. VA-ECMO was initiated due to progressive acidosis and hypoxemia, followed by a thoracotomy to temporize bleeding. Two additional thoracotomies were performed for recurrent hemothoraces. Due to the evolution of lung abscesses and sepsis, a pneumonectomy and tracheostomy were performed. Anticoagulation was held 6-24hours peri-operatively. His was maintained on ECMO for 9 days, required one circuit exchange, 62 units of PRBC, 12 units of FFP, 10 units of platelets and 20 units of cryoprecipitate during resuscitation. He was discharged to a rehab facility, tracheal collaring.

\section{Case \#4: Aspiration pneumonia/ ARDS}

A 48 year-old-male was found unconscious after a drug overdose with an aspiration event and subsequently developed ARDS. He was placed on VA ECMO for worsening respiratory acidosis and inability to oxygenate. While on ECMO, he had a disruption of his left femoral artery during positioning of the distal perfusion cannula requiring washout 
of a thigh hematoma, repair of femoral artery pseudoaneurysm and replacement of the distal perfusion catheter. Heparin was held 12 hours post-procedure. Post-operatively, this patient was noted to have gastrointestinal bleeding. Upper and lower endoscopies were performed without identification of the bleeding site. A push enteroscopy identified a jejunal bleeding site which was coagulated under the push endoscopy. The heparin was held 6 hours before gastrointestinal procedures and additional 6 hours after procedures. He remained on ECMO for 13 days, requiring no oxygenator exchanges, received 36 units of $\mathrm{PRBC}$ with 25 units of platelets. He was discharged to a rehabilitation facility tracheal collaring and tolerating tube feeds.

\section{Case \#5: Progressive viral pneumonia}

A 53 year old female was admitted with worsening respiratory distress due to a viral illness. She was placed on VV ECMO using the Avalon Elite Dual Lumen Cannula via right internal jugular vein. During cannulation, a pericardial effusion was diagnosed by transthoracic echocardiography, warranting immediate bedside pericardial window and operative repair of cannula-related right ventricular rupture. Two mediastinal washouts with packing were required until mediastinum closure at the time of her ECMO withdraw on day 9. Heparin was held 6-24 hours after each sternal intervention. Her resuscitation involved 42 units of PRBC, 14 units of FFP and 80 units of platelets. She was discharged home in 24 days with no oxygen requirements and adequate oral intake.

\section{DISCUSSION}


The first successful use of ECMO for respiratory support was reported in 1972 by Hill et al. ${ }^{4}$ An early clinical trial demonstrated poor survival and a large blood loss (>2.5L/day) in VA ECMO supported patients for respiratory failure. ${ }^{5}$ Despite improvements in circuit related technology, lung protection techniques and eligibility requirements, survival rates have not exceed $50-52 \%$ in this high risk population. ${ }^{6,7,8,9}$ Poor survival, as demonstrated, may be related to hemorrhagic complications, which are frequently seen in the ECMO population.

Bleeding is a common complication in ECMO supported patients contributing in part to the overall poor survival in this population. ${ }^{2}$ Contact of blood with ECMO circuitry leads to an intense pro-inflammatory and pro-thrombotic state, initiating the clotting cascade with activation of kininogen, kallikrein, factor XII, and platelets. This leads to activation of thrombin, factor XII and tissue plasminogen to counteract the clotting cascade. ${ }^{2} \mathrm{~A}$ decrease in platelets occurs, attributable to decreased production, increased consumption, sequestration, and dilution.The ongoing pro-coagulant and anticoagulant activity can lead to an imbalance of factors, resulting in thrombosis or hemorrhage and DIC. ${ }^{10}$

Due to the risk of clot formation with patients on ECMO, anticoagulation is necessary with the goal of preventing thrombosis while avoiding excessive bleeding. Historically, a fixed dose of heparin was employed as done with cardiopulmonary bypass, and anticoagulation with heparin was attained using a high therapeutic level of PTT $(60-80$ sec $)$ or activated clotting time $($ ACT $>180-240)$ to prevent thrombosis. ${ }^{10}$ Newer circulatory systems utilizing a heparin bonded tubing, a low pressure oxygenator and centrifugal pump allows for maintenance of anticoagulation on ECMO to be lower without thrombotic complications. VV ECMO may be maintained using heparin bonded tubing without administration of systemic heparin; however, in our experience clot 
formation in the cannulas and oxygenator is common often necessitating circuit exchange, increased expense and risk for systemic thrombosis or embolism. The ELSO Guidelines continue to recommend anticoagulation to prevent thrombotic complications. ${ }^{11}$ Our practice no longer uses ACT for titration of heparin due to unreliability $^{10}$; PTT levels are monitored with point of care testing. ${ }^{10,12}$

Sources of bleeding while on ECMO are varied and may be related to pre-ECMO procedures, may occur during ECMO cannulation, or as a result of prolonged cannulation, as evidenced by our series of patients. Bleeding complications related to pre-ECMO chest tube placement were common in this series. The majority of patients (Cases 1,2 and 3) had a chest tube placed prior to ECMO for barotrauma induced pneumothoraces and was subsequently anticoagulated during cannulation. These patients experienced chest wall hemorrhage at the site of chest tube insertion requiring surgical intervention within 24 hours of ECMO cannulation. In two of these cases, VATS were initially attempted to minimize bleeding risks when patients were anticoagulated, although definitive thoracotomies were eventually necessary in all three cases.

Cardiovascular structural damage may occur during placement of venous and/or arterial cannulas, as previously described by our group ${ }^{13}$. As observed in Case \#5, bedside placement of an Avalon catheter with the use of transthoracic echocardiography (TTE) may inadequately detect the guide-wire in the inferior vena cava during cannula placement which could result in myocardial injury. Transesophageal echocardiography may be required in conjunction with TTE to facilitate guide-wire and cannula placement. ${ }^{14,15}$ Patients who are stable should undergo fluoroscopic guidance for venous access. 
Management of the post-cardiotomy and post-thoracotomy open chest can be challenging, as experienced in this same Case \#5. Initiation of the low dose heparin protocol and cessation of heparin perioperative resulted in reduction in perioperative bleeding and allowed successful wound closure. Importantly there were no circuit related thrombotic or neurological complications in these patients.

Gastrointestinal bleeding from an arterio-venous malformation while on ECMO occurred in the patient in Case \#4 and has recently been described by our group. ${ }^{16}$ The etiology of this bleeding may be related to the lack of the arterial pulsatility in mesenteric perfusion. Options for identification of bleeding include upper and lower endoscopy and capsule studies. Our patient had a full diagnostic workup on ECMO while heparin was held for 48 hours; multiple sites of bleeding were found in the jejunum and push enteroscopy with coagulation was successful.

Although our small patient population may limit our results, we experienced no circuit related complications associated with the initiation or cessation of anticoagulation for bleeding complications, such as acute circuit thrombosis or systemic emboli, while following our anticoagulation protocol. The number of circuit exchanges due to poor oxygenation was minimal. The bedside circuit exchange was a simple procedure which did not require additional heparin bolus. No thrombotic complications were encountered during or after circuit exchange.

Success and survival in patients undergoing multiple surgical interventions while on ECMO have not been reported except in a single patient case report. ${ }^{17}$ Bleeding remains a major source of morbidity and mortality in the ECMO supported patient, particularly when surgical or endoscopic intervention is necessary. Successful control of bleeding and survivalwas attributed in part to advancements in ECMO circuitry, use of 
an anticoagulation protocol and an aggressive surgical approach to resolve bleeding in this high risk population. 


\section{CONFLICT OF INTEREST STATEMENT}

The authors have no conflicts of interest or financial ties to disclose. There was no outside funding for this work. 


\section{REFERENCES}

${ }^{1}$ Peek GJ, Mugford M, Tiruvoipati R, et al. CESAR trial collaboration. Efficacy and economic assessment of conventional ventilatory support versus extracorporeal membrane oxygenation for severe adult respiratory failure (CESAR): a multi-center randomized controlled trial. Lancet 2009; 374:1351-63.

2.Sidebotham D, McGeorge A, McGuinness S, et al. Extracorporeal membrane oxygenation for treating severe cardiac and respiratory failure in adults: part 2 technical considerations. J Cardiothorac Vasc Anesth 2010; 24: 164-72.

3. Conrad SA, Rycus PT, Dalton H. Extracorporeal life support registry report 2004. ASAIO J 2005; 51:4-10.

4. Hill JD, O’Brien TG, Murray JJ, et al. Prolonged extracorporeal oxygenation for acute post-traumatic respiratory failure (shock-lung syndrome). Use of the Bransom membrane lung. N Engl J Med 1972; 286:629-634.

5.Zapol WM, Snider MT, Hill JD, et al. Extracorporeal membrane oxygenation in severe acute respiratory failure. A randomized prospective study. JAMA 1979; 242:21932196.

${ }^{6 .}$ ARDSnet. Ventilation with lower tidal volumes as compared with traditional tidal volumes as compared with traditional tidal volumes for acute lung injury and the acute respiratory distress syndrome. N Engl J Med.2000;342:18:1301-8.

${ }^{7}$ Brodie D, BAcchetta M. Extracorporeal Membrane Oxygenation for ARDS in Adults. N Engl J Med. 2011;365:1905-1914. 
${ }^{8 .}$ Brogan TV, Thiagargajan RR, Rycus PT, et al. Extracorporeal membrane oxygenation in adults with severe respiratory failure: a multi-center database. Intensive Care Med. 2009;35: 2105-2114.

${ }^{9}$ Hemmila MR, Rowe SA, Boules TN, et al. Extracorporeal life support for severe acute respiratory distress syndrome in adults. Ann Surg. 2004;240:595-607.

${ }^{10 .}$ Oliver W. Anticoagulation and coagulation management for ECMO. Seminar Cardiothoracic Vascular Anesth 2009;13: 154-75.

${ }^{11 .}$ ELSO Guidelines for Cardiopulmonary Life Support, Extracorporeal Life Support Organization, Version 1.1. Report. Univsity of Michigan. April 2009. www.elso.med.umich.edu

${ }^{12 .}$ Reed RC, Rutledge JC. Laboratory and clinical predictors of thrombosis and hemorrhage in 29 pediatric extracorporeal membrane oxygen non-survivors. Pediatr Dev Pathol 2010;13:385-92.

${ }^{13 .}$ Hirose H, Yamane K, Marhefka G, et al. Right ventricular rupture and tamponade caused by malposition of the Avalon cannula for venovenous extracorporeal membrane oxygenation. J Cardiothorac Surg. 2012; 7:36.

${ }^{14 .}$ MacLaren G, Combes A, Bartlett RH. Contemporary extracorporeal membrane oxygenation for adult respiratory failure: life support in the new era. Intensive Care Med. 2012:38:210-220.

${ }^{15}$ Bermudez CA, Rocha RV, Sappington PL, et al. Initial experience with single cannulation for venovenous extracorporeal oxygenation in adults. Ann Thorac Surg. 2010:90:3; 991-5. 
${ }^{16 .}$ Sarosiek K, Hirose H, Pitcher H, et al. Adult ECMO and gastrointestinal bleeding from small bowel arteriovenous malformations: A novel treatment using spiral enteroscopy," has been accepted for publication. Accepted for publication for $J$

Thorac Cardiovasc Surg.

17.Marasco SF, Preovolos A, Lim K, et al. Thoracotomy in adults is associated with uncontrollable bleeding. Perfusion 2007;22:23-

26. 